\title{
ANÁLISIS Y ELIMINACIÓN: UNA MÓDICA DEFENSA DE QUINE ${ }^{1}$
}

\author{
THOMAS M. SIMPSON \\ Consejo Nacional de \\ Investigaciones Científicas \\ de la Argentina
}

Coffa ha caracterizado la elucidación como un proceso de tres etapas:

1) Identificación del concepto a elucidar (explicandum), que posee un grado mayor o menor de vaguedad.

2) Formulación de los criterios de adecuación o condiciones que debe satisfacer cualquier concepto que pretenda ser una elucidación del primero; estas condiciones constituyen un análisis parcial de explicandum.

3) Formulación del explicatum, o sea del concepto que satisface tales condiciones.

Conviene prestar atención al hecho de que el análisis es aquí sólo parte del proceso de elucidación, cuando lo más usual es hablar simplemente del proceso de 'análisis' en general, proceso en el cual el paso 2 contiene los análisis parciales con los que debe concordar el análisis completo. El punto es fundamental, pero no me detendré en él por el momento; baste decir que "elucidación" es una versión castellana de "explication", término puesto en circulación por Carnap para distinguir su pro-

${ }^{1}$ (Septiembre de 1975). El texto que sigue fue leído en la Sociedad Argentina de Análisis Filosófico (SADAF) el 11 de agosto de 1972, en respuesta al trabajo de Alberto Coffa "Dos concepciones de la elucidación filosófica", cuya versión modificada se publica en el presente número de Crítica. Aunque no he introducido cambio alguno, creo que estos comentarios poseen cierto valor autónomo y que el diálogo con Coffa es todavía inteligible. Sólo me permití agregar cinco notas de pie de página, a modo de tímido 'aggiornamiento'. 
pia concepción sobre la naturaleza del análisis filosófico. (De allí la correspondencia con "explicandum" y "explicatum"; nosotros debiéramos decir, quizás, "elucidandum" y "elucidatum"). Pero algunos autores usan ambos términos en forma intercambiable; Quine (Word and Object, p. 259) dice simplemente: "el análisis filosófico, la elucidación", sin darle a la última expresión un sentido técnico especial. Pero volveremos después sobre este punto.

Un ejemplo simple de este proceso es el siguiente: Deseamos elucidar la noción de 'conocimiento'; pero como la palabra es ambigua, identificamos el objeto de nuestra elucidación diciendo que se trata del concepto expresado en contextos como "Juan sabe que la luna es redonda", pero no en contextos como "Juan sabe manejar" o "Esto sabe a limón"; se trata, pues, de lo que se denomina "conocimiento proposicional", por el hecho de que el objeto del conocimiento se presenta gramaticalmente como lo expresado por una oración sustantivada. El paso 2 consiste en establecer, por ejemplo, que un explicatum adecuado debe conservar la verdad de la siguiente implicación: "Si $X$ sabe que $p$ entonces $p$ es verdadera", o sea que el conocimiento proposicional implica verdad. Coffa insiste en que este enunciado no es una mera estipulación, sino una afirmación verdadera o falsa, y que si es verdadera es necesaria en virtud del significado ordinario de "saber que" en el contexto indicado; es precisamente este hecho el que hace posible que una elucidación sea errónea.

El tercer paso es dar al explicatum, que podría ser, aproximadamente, el que examina Platón en el Teeteto: $X$ sabe que $p$ si y sólo si: a) $X$ cree que $p$; b) $X$ tiene buenas razones para creerlo; e) $p$ es verdadera. No es un detalle sin importancia que esta definición fuera rechazada por Platón, y que nadie haya podido remendarla satisfactoriamente en los últimos 2500 años, pese a que cualquier persona con un dominio básico del lenguaje sabe usar de manera correcta la expresión "saber que" y en- 
tiende perfectamente lo que se le dice cuando oye que Juan sabe que la luna es redonda.

Ahora bien, a este modelo de elucidación se ajustan, según Coffa, las elucidaciones practicadas por Tarski en relación con diversos conceptos; pero esta afirmación es al menos parcialmente discutible. Las citas del propio Tarski son ilustrativas: dice, por ejemplo, que su definición de 'Verdad' se propone "captar el significado real (the actual meaning) de una noción vieja"; y el mismo objetivo: "expresar el significado real de un término", aparece en relación con su definición del concepto de 'conjunto definible de números reales'. En este último caso compara su tarea con la de los geómetras que establecieron por primera vez el significado de los términos "movimiento", "línea", "superficie" y "dimensión". En cuanto a su definición de "concepto definible" (Logic, Mathematics and Metamathematics, p. 302), logra el desideratum de probar matemáticamente que la extensión del explicatum es la misma que la del explicandum, proeza imposible de realizar en el caso del concepto de 'Verdad', que le dio fama en el mundo filosófico, o de cualquier otro concepto empírico.

Oponiendo esta concepción a la de Quine, Coffa dice que la relación entre el explicatum y el explicandum "es aquí directa: el segundo es una clarificación del primero". En cierto sentido esto es evidente, si 'clarificar' es presentar un explicatum en las condiciones enumeradas antes; por otra parte, si tomamos la declaración de Tarski en forma literal, la relación parece presentarse como la más directa imaginable: la de una identidad, que es la forma tradicional de entender un análisis de conceptos, filosóficos o no. Pero esto no concuerda con el modelo de elucidación defendido por Coffa, que sólo exige una identidad parcial. Me detendré, pues, en este punto, que se conecta con la distinción entre 'elucidación' y 'análisis', a la que me he referido anteriormente.

En primer lugar, el explicandum no es simplemente una expresión que no exhibe a la luz del día los componentes concep- 
tuales que pone de manifiesto el explicatum, o sea un término cuyo pecado es la discreción, y cuyos secretos revela el explicatum: en rigor, el problema del análisis filosófico es que el explicandum posee grados variables de vaguedad, y no hay modo de establecer los límites exactos de su aplicación correcta; y si bien los criterios de adecuación - cuando son verdaderos - sirven para explicitar el núcleo identificable de su significado intuitivo, queda un resto de penumbra que la elucidación debe completar; pero ¿de qué manera? Aquí es donde intervienen los aspectos convencionales y pragmáticos, derivados del hecho de que diversos explicata pueden satisfacer los mismos criterios de adecuación, y ser, por lo tanto, igualmente correctos desde este punto de vista. Sin embargo, siendo diferentes entre sí, no es posible que todos expresen 'el significado real' del explicandum. Si usamos la palabra "significado" en un sentido fuerte, entonces los diversos explicata que constituyen una clarificación del mismo concepto poseen sin embargo significados distintos; no pueden ser sinónimos, como es fácil mostrar. Por otra parte, sólo mediante un uso débil de esta palabra se puede pretender - como lo hace Tarski- que las definiciones geométricas de 'Círculo', 'Superficie' y 'Dimensión', que introducen conceptos y entidades cuya relación con la experiencia cotidiana es más que problemática, captan "el significado real" de estos términos en el lenguaje ordinario. En estos casos, clarificar un concepto es construir un concepto nuevo, con el margen de arbitrariedad que ello implica, pues el explicatum debe permitir o excluir claramente su aplicación a casos en relación con los cuales el término original se mantiene en silencio. Esto es reconocido por Tarski mismo en otro texto, en el cual, después de comparar el problema de la definición de 'conjunto definible de números reales' con el de definir 'superficie' y ‘dimensión' agrega que

en geometría el problema era volver precisas las intuiciones parciales adquiridas empíricamente en la vida diaria, intuiciones que por 
su propia naturaleza son vagas y confusas. Aquí tratamos con intuiciones más claras y concientes, de naturaleza lógica... Los geómetras se vieron obligados a elegir uno entre varios sentidos incompatibles, pero aquí la arbitrariedad en establecer el contenido del término se reduce casi a cero (loc. cit., p. 112).

Y en el texto de Menger sobre la definición de 'dimensión', citado por Coffa, se dice que con respecto a los objetos "no conocidos o no tratados en el lenguaje ordinario una definición (elucidación) no puede dejar de ser arbitraria". El mismo Coffa observa, al comentar el proceso tarskiano de elucidación, que al final del proceso "hemos clarificado, rigorizado y quizás extendido el "explicandum" (la bastardilla es mía). Pero si la extensión de un concepto - el explicatum - es mayor y difiere de manera arbitraria de otro concepto - el explicandum - entonces no puede tratarse del mismo concepto, pues la extensión de un término está determinada unívocamente por su significado (o sea por el concepto que expresa).

Es casi seguro que Coffa está también de acuerdo con todo esto; pero quizás su terminología no sea suficientemente cautelosa a este respecto, pues reprocha a Quine que en su concepción del análisis filosófico "no hay un objeto común de referencia para las expresiones usadas antes y después de la elucidación"; y si no lo interpreto mal, ese objeto común no puede ser otro que el concepto elucidado. Pero, como acabamos de ver, tal objeto común en realidad no existe. ${ }^{2}$ En conse-

2 (Septiembre 1975). En la versión actual de su trabajo, Coffa considera las dificultades que presenta para el tarskismo la "cosificación de conceptos y significados" y encuentra una esperanza de salvación en las teorías semánticas de Sellars: "Si no hubiera forma de evitar la idea errónea del significado que reverbera ... en algunos de los textos ... en que Carnap se presenta como tarskiano, habría que concluir que el tarskismo es incapaz de dar a sus buenas intenciones un fundamento semántico adecuado. Felizmente ... la obra de Sellars parece indicar que es posible desarrollar una teoría del concepto basada en el rechazo de una concepción 'cosista' del significado". Sin embargo, me temo que Sellars no sea el hombre que Tarski necesita para resolver el 
cuencia, y consideradas las cosas en forma estricta, si bien con una dosis innegable de fanatismo semántico, al elucidar no captamos "el significado real" de un término, sino que reemplazamos un concepto por otro: Elucidación es eliminación, para decirlo con la incisiva fórmula de Quine. Naturalmente, este eslogan epigramático tiene una intención polémica que se presta al equívoco, y no me inclino a aceptar el alcance más general que pretende conferirle Quine cuando considera problemas como el de la relación entre lo mental y lo físico. Pero es importante advertir contra quién está dirigida primariamente su fórmula nihilista, si deseamos captar su 'significado real' y la parte de verdad que contiene. Su enemigo es la concepción tradicional del análisis como una ecuación conceptual, concepción que genera la llamada paradoja del análisis, que puede formularse brevemente así: $\mathrm{Si}$ "El concepto $A=$ el concepto $B$ " es un análisis correcto, significa lo mismo que "El concepto $A=$ el concepto $A$ "; y si no significa lo mismo que esta identidad trivial, entonces el análisis es falso. Carnap se negó a elegir entre la trivialidad y la falsedad, y optó por romper el círculo modificando la noción de análisis filosófico: para distinguirla de la noción tradicional la llamó "explication" (elucidación). Es en este punto en el que Quine coincide con Carnap. Para que sus motivos se vean más claramente, citaré un texto de este último que me parece de particular interés:

El problema de la elucidación — dice Carnap- es característicamente distinto de los problemas científicos ordinarios (lógicos o

problema esencial que estamos considerando: el de la pretendida identidad entre el analysandum y el analysans; según el mismo Sellars, "un concepto no puede ser idéntico al concepto del cual es el análisis" (W. Sellars, "The Paradox of Analysis: A Neo-Fregean Approach", en Analysis, Vol. 24, No. 2, enero 1964). En este punto, al menos, Sellars está cerca de Quine. El lector hallará un examen detallado de esta cuestión en mi artículo "Sobre la solución fregeana de Morton White a la paradoja del análisis", incluido en Thomas M. Simpson, Semántica Filosófica: Problemas y Discusiones, Siglo XXI Editores, Buenos Aires-Madrid, 1973. 
empíricos), donde, en condiciones favorables, tanto el datum como la solución se hallan expresados en términos exactos; por ejemplo, “¿cuál es el producto de 3 y 5 ?”, "¿qué ocurre cuando una corriente eléctrica atraviesa el agua?". En un problema de elucidación el datum, o sea el explicandum, no está dado en términos exactos; si lo estuviera, no sería necesaria elucidación alguna. Puesto que el datum es inexacto, el problema mismo no se halla formulado en términos exactos, y sin embargo se nos pide que demos una solución exacta. Esta es una de las características desconcertantes del proceso de elucidación. Se sigue que, cuando se propone una solución para un problema de elucidación, no podemos decidir de manera exacta si es correcta o adecuada. Hablando estrictamente, la cuestión de si la solución es correcta o errónea no tiene ningún sentido, porque no existe ninguna respuesta tajante. Debe preguntarse más bien si la solución propuesta es satisfactoria, más satisfactoria que otras, etcétera.

No me propongo defender in toto estas formulaciones extremas, ni queda tiempo para examinar en detalle sus diversos aspectos; sólo me interesa aquí el problema planteado por la inexactitud del explicandum, que hace imposible que la elucidación proporcione una identidad intensional. En cuanto a la negación de sentido a la pregunta sobre el carácter 'correcto' de la elucidación, podría dársele al texto una interpretación ecuánime suponiendo que Carnap está pensando en la concepción tradicional de 'análisis correcto', que exige aquella identidad imposible y postula, por lo tanto, la existencia de un único explicatum: adecuado; pues para expresarnos en una forma ligeramente paradójica, es obvio que en un sentido relativo de "corrección" cualquier explicatum es absolutamente correcto si satisface los criterios de adecuación en el caso de que éstos sean verdaderos, y de alguna manera suficientes.

Sin embargo la cuestión no es simple, como lo ilustran — según veremos - las desconcertantes oscilaciones del propio Carnap.

Coffa ha citado un texto curioso en el que Carnap adopta la concepción tradicional del análisis de la manera más ingenua 
posible, pues nos viene con el cuento increíble de que nadie conocía antes de Frege "el significado real" de las expresiones numéricas. Llamemos a este Carnap esencialista Carnap* (Carnap Estrella) para conectarlo con el cielo platónico. Es precisamente contra las concepciones de Carnap Estrella que Carnap y Quine sostienen una noción de análisis filosófico en el que sólo tiene cabida una concordancia parcial entre el explicandum y el explicatum; y vale la pena recordar que Husserl y Peano rechazaron la definición fregeana de Número natural por no ser intensionalmente idéntica al concepto intuitivo, o sea porque el explicatum no captaba 'el significado real' de la expresión "número natural", y, en particular, de los numerales específicos. Es que, como bien lo advirtió Carnap, sólo un iluso como Carnap Estrella pudo pensar que el análisis del significado en sentido estricto podría conducirnos a descubrir que el número 2 es precisamente la clase de todos los conceptos $f$ que se caracterizan por lo siguiente: existen objetos $x, y$ tales que $f x$ y $f y$, y cualquiera que sea $z, f z$ si y sólo si $z=x$ o $z=y$. Lo más notable del caso es que Frege mismo consideró la posibilidad de una definición diferente, en que la clase de conceptos es reemplazada por un concepto de segundo nivel, definición que difiere intensionalmente de la anterior tanto como puede diferir una clase de una propiedad, y que Carnap adoptó en su libro Meaning and Necessity en lugar de la definición original de Frege, donde - según Carnap Estrella - está explícito "el significado real". Parece razonable sostener que si alguien no sabe que "Soltero" significa No casado, o que "Padre" significa Progenitor Masculino, no posee un dominio completo del lenguaje; pero es grotesco sugerir que no domina el lenguaje - en el sentido de no conocer los significados - porque 'ignora' (o mejor, no cree, y con razón) que un número es realmente una clase de conceptos equinumerosos. Por eso dice Carnap que en una elucidación "no se requiere que el explicatum posea el mismo significado que el explicandum" (Meaning and Necessity, p. 8). Y Quine, como un eco fiel: "No pretendemos esta- 
blecer una sinonimia; no pretendemos volver claro y explícito lo que los usuarios de una expresión no clara tenían inconscientemente en su cabeza. No exponemos a la luz significados ocultos, como podrían sugerir las palabras 'análisis' y 'elucidación"” (Word and Object, p. 258). Esto, naturalmente, es lo que no se hace; pero ¿qué es lo que sí se hace según Quine? Antes de considerar el tema - y haré un esfuerzo por defenderlo "hasta la horca exclusive", para citar una frase feliz de Rabelais- agregaré algo más sobre Tarski y su uso de la palabra "significado".

Tarski habla del 'significado real', pero es casi seguro que sus definiciones hubieran sido rechazadas por Husserl aun en los casos afortunados en los que se puede probar lógicamente la identidad extensional de ambos términos de la definición, para no mencionar aquellos casos en que, por tratarse de conceptos empíricos, esto es literalmente imposible. Recordemos, al pasar, que Kant sostenía que los conceptos de clases naturales no son definibles en sentido estricto; su extensión exacta no puede ser determinada.

Este punto es fundamental en relación con el problema del análisis, pero temo que la noche no alcance, de modo que no me detendré por ahora en él. En cuanto a la definición del concepto de 'verdad' dado por Tarski, que Quine considera un paradigma de análisis filosófico, y que según Tarski capta 'el significado real' contenido en el uso ordinario del término, es evidente para mí - y también para Quine - que tal sinonimia no existe. Cuando decimos esto pensamos en una noción fina de sinonimia que suele vincularse con la reformulación adecuada de una oración en el discurso indirecto y con la noción de traducción correcta, noción que es más fuerte que la equivalencia lógica y se vincula también con la analiticidad.

Aquí hay varios problemas enredados que no puedo tratar, pero mencionaré brevemente: el texto de Quine alude a los significados "ocultos en la mente de los hablantes", y niega que una elucidación deba sacarlos de allí para exhibirlos; pero esta noción psicológica de significado no es necesaria para la tesis de 
Quine. La negación de la sinonimia analítica puede sostenerse independientemente de ella, considerando, por ejemplo, que los significados no están en la mente del hablante, del mismo modo que las leyes de la mecánica no están en la mente del ciclista que maneja de acuerdo con ellas; así como la mecánica proporciona la teoría de lo que el ciclista hace, la elucidación - podría sostenerse - ofrece la teoría de la práctica verbal del hablante, extrayendo de ella los criterios de aplicación que no están en su mente pero gobiernan su conducta lingüística. Aun así, prescindiendo de toda referencia a contenidos introspectivos, Quine podría reformular su protesta diciendo que los usos lingüísticos - para emplear una palabra manoseada y ambigua- constituyen una red difusa cuyas lagunas deben ser llenadas por la teoría y que ninguna fórmula puede reflejar con exactitud. Aquí el formalismo de Quine puede apoyarse eficazmente en el antiformalismo del segundo Wittgenstein.

Paso ahora a la concepción positiva de Quine. Deseo recalcar tres puntos, de la manera más apretada que me sea posible:

1. ¿Qué son, después de todo, los criterios de adecuación? En cierto modo, son precisamente aquellos contextos que según Quine "merecen salvarse" y que él califica de claros y útiles; no importa que no los considere verdades necesarias, puesto que no cree en ellas. Pero en la práctica la situación es la misma: el explicatum: debe concordar con ellos. Así ocurre en el caso del criterio de adecuación de Tarski para el concepto de verdad, que debe deducirse de la definición. Quizás no sea entonces demasiado justo afirmar - como lo hace Coffa - que en la concepción de Quine el explicatum se vincula con el explicandum "de maneras inexplicables".

2. Sería ecuánime distinguir en Quine dos aspectos:

a) La tesis nihilista de que "elucidación es eliminación", la cual puede defenderse del modo indicado antes. Es claro, sin embargo, que para Quine la elucidación es tanto eliminación como preservación, al menos en la medida en que admite contextos privilegiados cuya validez "merece salvarse", como el 
criterio de identidad de pares ordenados y el criterio de adecuación de Tarski.

b) Su generalización, a partir de casos específicos relativamente simples, de los vagos criterios de "utilidad", "cumplimiento de los mismos propósitos", etc., a situaciones filosóficas más complejas, como la elucidación del conocimiento o la inducción.

En el punto b) me inclino a concordar con Coffa. ${ }^{3}$ Entre los casos simples incluyo la definición de número natural, por tratarse de un concepto que pertenece a su vez a un sistema conceptual bien definido, de modo que el criterio de utilidad no es otro que el de preservar las relaciones que rigen en tal sistema, lo que permite construir otros sistemas isomórficos que no exigen siquiera el requisito de identidad extensional. Nadie ha visto nunca un número: sólo sabemos que dos más dos son cuatro; que cinco es menor que ocho, etc.; no es de extrañar, entonces, que en una elucidación de la aritmética a la manera clásica nos conformemos con elegir - o inventar - representantes de los números naturales entre los que resulten válidas las relaciones correspondientes. El verdadero problema filosófico comienza en rigor en este punto, en lugar de terminarse, como parece sugerirlo Quine.

Con respecto a conceptos como 'verdad' y 'conocimiento', que no forman parte de un sistema teórico definido, los criterios de "utilidad" y "función" esgrimidos por Quine poseen un valor dudoso: la única utilidad concebible de una elucidación adecuada de "conocimiento" es ayudarnos a comprender en qué consiste. ${ }^{4}$

c) La práctica filosófica del mismo Quine no se ajusta a sus reglas explícitas. Así, su rechazo de las entidades intensionales por carecer de condiciones claras de identidad se basa en un

${ }^{3}$ (Septiembre 1975). Esta coincidencia es limitada: no abarca, naturalmente, el requerimiento de una identidad entre el analysans y el analysandum.

${ }^{4}$ (Septiembre 1975). Sí, pero, ¿̇en qué consiste comprender? 
criterio filosófico acerca de la confiabilidad de una ontología que no puede reducirse a la mera utilidad. De lo contrario debería aceptar la semántica de Frege-Church, que permite salvar la validez general de la ley de Leibniz: pero Quine la rechaza guiado por su olfato metafísico. Otro ejemplo es su análisis de la creencia transparente o de re, que pretende distinguir mediante una notación nueva un uso de "creer" que aparece mezclado con otros en el lenguaje cotidiano; no importa que Quine no hable del significado, sino de contextos útiles. En este caso el contexto útil sería descrito platonísticamente como aquel en el cual se expresa un concepto de 'creencia' de fundamental importancia en la comunicación humana.

Tendría que hablar ahora del punto que más me interesa: el argumento del caso paradigmático, que según Coffa subyace a las concepciones del análisis filosófico que se esfuerza en demoler. ${ }^{5}$ Me gustaría defender dos cosas:

1) La idea de que, contrariamente a la doctrina común, hay una primacía de la extensión sobre la intensión, y, en consecuencia,

2) La legitimidad de una forma débil - pero importantedel argumento del caso paradigmático.

En cuanto a la versión fuerte divulgada por Ryle y otros filósofos del lenguaje ordinario, comparto el aborrecimiento que le inspira a Coffa. Lamentablemente, el examen de estas cuestiones sobrepasa el tiempo que me fue conferido; y como dijo Wittgenstein en el célebre y misterioso aforismo con que termina el Tractatus, "cuando a uno no lo dejan hablar, lo mejor es callarse".

${ }^{5}$ (Septiembre 1975). Como puede comprobarlo el lector, el examen de esta cuestión no figura en la versión final del artículo de Coffa. 


\section{SUMMARY}

In this paper I intend to appraise Quine's dictum: "Explication is Elimination" and to defend it from Coffa's attack (cf. this issue, pp. 43-67, for Coffa's position).

With respect to Coffa's characterization of explication (cf. Coffa's characterization of the Tarski-Kreisel conception of explication, which I take him as accepting, on pp. 48-54), I want to hold in the first place that the explicandum is not simply an expression which does not exhibit in daylight the conceptual components which the explicatum makes evident; strictly speaking the problem of philosophical analysis is that the explicandum possesses varying degrees of vagueness and there's no way of establishing the exact limits of its correct application; even if the adequacy criteria - when true- are useful to make the identifiable nucleus of its intuitive meaning explicit, a shady part remains which the explication has to complete somehow.

What I hold is that to clarify a concept is to build up a new one, with a certain margin of arbitrariness, since the explicatum clearly has to allow or to exclude its application to those cases about which the original term said nothing. Coffa himself observes, in commenting on the Tarskian process of explication, that by the end of it "the explicatum has been clarified, made more rigorous and it may also have been expanded" (the emphasis is mine). But if the extension of a concept - the explicatum - is larger and in an arbitrary way differs from that of another concept - the explicandum-, then we are not dealing with the same concept since the extension of a term is univocally determined by its meaning (i.e. by the concept it expresses).

Coffa says against Quine that in this author's conception of philosophical analysis "there's no common object of reference for those expressions used before and after the explication"; and if I take him right, that common object can be no other than the explicated concept. But in fact, as we have just seen, that common object does not exist. So, strictly speaking, what we do in explicating a term is not to apprehend its "real meaning" but rather to replace one concept for another: "Explication is elimination". Now, so as not to take Quine's dictum in a wrong way, one has to be clear against whom this formula 
is directed. It can be taken as a polemic invective against the traditional conception of analysis which gives rise to the paradox by the same name. Carnap rejected the paradox by modifying the notion of philosophical analysis; to distinguish it from the traditional one he called his "explication". It is at this point that Quine and Carnap do coincide.

As to Coffa's reading of Carnap as a Tarskian, I must say that Coffa selected a very curious passage in which Carnap adopted the traditional conception of analysis in the most candid way imaginable. And it is against this candid Carnap that Carnap himself and Quine jointly maintain a view of philosophical analysis in which it is only possible to accept a partial agreement between the explicandum and the explicatum. Carnap, in Meaning and Necessity (p. 8), says that in an explication "it is not required that an explicatum have, as nearly as possible, the same meaning as the explicandum". And Quine, as a faithful echo, says (W \& O, p. 258): "We do not claim synonymy. We do not claim to make clear and explicit what the users of the unclear expression had unconsciously in mind all along. We do not expose hidden meanings as the words 'analysis' and 'explication' would suggest...". These are all negative points. Which are the positive ones for Quine?

I want to emphasize the following points in a most compact way:

1. Concerning the adequacy criteria, one can say that in a sense, they are precisely those contexts which, according to Quine, are "worth saving" and which he qualifies as clear and useful. So it may not be too fair to state — as Coffa does - that within the Quinean conception the explicatum is related to the explicandum "in a way difficult to explain".

2. It could be fair to distinguish two aspects in Quine:

a) His thesis that "explication is elimination", which can be defended in the above-mentioned way. But one can also make the point that for Quine an explication not only eliminates but also preserves those distinguished contexts "worth saving".

b) His generalization of the vague criteria of "usefulness", "fulfillment of same purposes", etc., starting from relatively simple specific cases to more complex philosophical situations, as the explication of knowledge or induction. 
At this point I feel I agree with Coffa. With respect to concepts as "truth" and "knowledge" which are no part of a defined theoretical system, the Quinean criteria of 'use' and 'function' have a dubious value: the only thinkable utility of an adequate explication of "knowledge" is to help us understand what it is.

c) Quine's philosophical practice itself does not live up lo his explicit rules. For example, his rejection of intentional entities because of a lack of clear identity conditions is based on a philosophical criterion about the reliability of an ontology which cannot be reduced to mere utility. Otherwise he ought to accept the Frege-Church semantics which lets him have the general validity of Leibniz law: but Quine rejects it due to his metaphysical keenness.

Summary by José A. Robles 